\title{
A simplified model for the shielding of fire thermal radiation by water mists
}

\author{
Leonid A. Dombrovsky ${ }^{1 *}$, Siaka Dembele ${ }^{2}$, and Jennifer X. Wen ${ }^{3}$ \\ ${ }^{1}$ Joint Institute for High Temperatures, Krasnokazarmennaya Str. 17A, NCHMT, Moscow 111116, Russia \\ ${ }^{2}$ Fire, Explosion and Fluid Dynamics Research Group, School of Mechanical and Automotive Engineer- \\ ing, Kingston University, London SW15 3DW, UK \\ ${ }^{3}$ Warwick FIRE, School of Engineering, University of Warwick, Coventry CV4 7AL, UK
}

\begin{abstract}
A solution for the complete problem of attenuation of fire radiation by water mist is presented. This solution is based on simplified approaches for the spectral radiative properties of water droplets, the radiative transfer in the absorbing and scattering mist, and transient heat transfer taking into account partial evaporation of water mist. A computational study of the conventional model problem indicates the role of the main parameters and enables one to formulate some recommendations to optimize possible engineering solutions. The method developed is also applied to more realistic case study of a real fire. It is suggested to decrease the size of supplied water droplets with the distance from the irradiated surface of the mist layer. The advantage of this engineering solution is confirmed by numerical calculations. Potential possibility of microwave monitoring of water mist parameters is analyzed on the basis of Mie theory calculations.
\end{abstract}

Keywords: Fire radiation; Water mist; Radiative transfer; Heat transfer; Computational model.

\footnotetext{
* Corresponding author: Tel.: +7 9104080186

E-mail address: 1dombr@yandex.ru (L.A. Dombrovsky)
} 


\section{Nomenclature}

\begin{tabular}{|c|c|c|c|}
\hline$a$ & radius of droplet & $\kappa$ & index of absorption \\
\hline$A$ & attenuation parameter introduced by & $\lambda$ & wavelength \\
\hline & Eq. (17) & $\mu$ & cosine of an angle \\
\hline & specific heat capacity & $\rho$ & density \\
\hline I & drag coefficient & $\sigma$ & scattering coefficient or electrical con- \\
\hline $\mathrm{v}$ & volume fraction of droplets & & ductivity \\
\hline & height of the mist curtain & $\sigma_{0}$ & Stefan-Boltzmann constant \\
\hline & thermal conductivity & $\omega$ & scattering albedo \\
\hline & latent heat of evaporation & & \\
\hline & complex index of refraction & Subs & cripts and superscripts \\
\hline 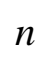 & index of refraction & a & absorption \\
\hline & radiative flux & $\mathrm{d}$ & droplet \\
\hline$O$ & efficiency factor of absorption or scat- & $\mathrm{e}$ & external \\
\hline & tering & el & electrical \\
\hline$R$ & reflectance & $\mathrm{f}$ & flame \\
\hline 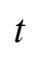 & time & $\mathrm{g}$ & gas \\
\hline$T$ & temperature & $\mathrm{h}$ & hemispherical \\
\hline$u$ & velocity & $\mathrm{n}-\mathrm{h}$ & normal-hemispherical \\
\hline$W$ & absorbed radiation power & rel & relaxation \\
\hline$\lambda$ & diffraction parameter & s & scattering or static \\
\hline$y$ & horizontal coordinate & $\mathrm{t}$ & transmitted \\
\hline$z$ & vertical coordinate & tot & total \\
\hline & & $\operatorname{tr}$ & transport \\
\hline & k symbols & $\mathrm{w}$ & water \\
\hline$\alpha$ & absorption coefficient & $\lambda$ & spectral \\
\hline & dielectric constant & & \\
\hline & dynamic viscosity of gas & & \\
\hline
\end{tabular}

\section{Introduction}

Since the ratification of the Montreal protocol in 1987, phasing out halon agents due to their negative environmental impacts, the use of water sprays and mists in fire protection has gained momentum. Water mist is defined according to the NFPA as sprays in which $99 \%$ of the volume is in droplets with diameters less than 1000 microns. Water spray/mist systems can be used for the dilution of toxic releases [1]. The scope of the present study is its fire application. There are two main strategies for using water sprays/mists in fire protection. In the first one, the intention is to extinguish or control the fire by applying the spray directly onto the fire source. Such applications have been well reviewed in [2] and considered also in [3]. 
In the second application strategy where there is no direct contact between the fire source and spray, the curtain of spray/mist is used as a radiation attenuation shield to protect potential targets which could be equipments or human beings [3]. The present study is concerned with such radiation shielding applications of water mist curtains. In the process industries, spray/mist curtains provide an effective mean to protect flammable targets (e.g. storage tanks) in the event of fires. They could also serve as protection against fire radiation for personnel during evacuation on-board carrier and chemical ships during maritime transport [4]. In some countries, fire engines used by firefighters to combat forest fires are fitted with water spray curtains as emergency personnel protection. Water spray curtains can also be employed as compartmentation to protect people in fire events [5].

Research on water spray/mist shielding has received considerable attention in the past two decades. Although the main mechanisms of radiation attenuation by a two-phase water spray have been identified as absorption and scattering by droplets and absorption by the gas phase (mainly water vapor), a rigorous model that account for the coupled radiation, heat and mass transfers in the spray is complex to develop and is too involved computationally. Such models are important to better design and optimise water sprays and mists for reliable and costeffective solutions.

The bulk of the literature on water spray/mist curtain shielding has been devoted to radiation modelling by uncoupling it from other phenomena, in order to predict the transmittance and attenuation of the curtain. Obviously, the Beer-Lambert law used in [6] is inapplicable for the transmittance calculations in the problem under consideration. Therefore, the two-flux model was employed in more recent papers [7-10] for the transmittance calculations. These studies show that smaller droplets in high concentration provide better attenuation of the spray. However the important phenomena such as mass transfer and droplets evaporation were not considered in these papers.

More detailed description of radiative transfer based on the Discrete Ordinates Method (DOM), Finite Volume Method and even Monte Carlo simulation were also used in computational studies of water mists [11-17]. Some of these studies have coupled the radiation, heat, mass and momentum transfer in sprays using the combined Eulerian-Lagrangian approach for both dynamic and thermal non-equilibrium of droplets and ambient gas. However the complexity of this approach is an obstacle to their widespread use.

The current literature clearly shows the advances in modelling and improving the understanding of water spray/mist curtain in fire radiation mitigation. However these models are mainly employed by the research community and are rarely used in the water mist industry which is currently developing fast and needs such tools. The complexity and computing cost 
are clearly two major obstacles for the application of current methods. There is a need nowadays to develop engineering models for water mist curtain, which retains the physics of the problem and at the same time offers acceptable computing cost. The present study aims to achieve such a goal.

The objectives of the present paper are as follows: (1) to develop a simplified but complete model for the combined heat transfer processes in a semi-transparent layer of water droplets used as a shield for infrared radiation of fires, (2) to study computationally the role of the main parameters of water mist and to give preliminary recommendation on possible optimization of engineering solutions for fire protection, (3) to present a numerical solution for realistic case study, (4) to suggest possible principal approach to the microwave monitoring the mist parameters taking into account the effect of both the size and temperature of water droplets on absorption and scattering of the microwave radiation by the mist layer.

The methodology of the present paper is based on a combination of a set of approximate 1D solutions for the radiative transfer problem and a simplified heat transfer model for heating and evaporation of water droplets. A relatively small absorption of radiation by water vapor, which is generated by partially evaporating water droplets, is neglected in a simplified model. The analysis of the analytical solution for radiative heat transfer through the mist layer makes it possible to suggest a decrease in the size of supplied water droplets with the distance from the irradiated surface of the mist layer. Subsequent numerical calculations for more realistic case study of fire protection confirm the advantages of the suggested engineering solution.

An analysis of possible microwave monitoring of water mist parameters is also given in the paper. The calculations based on the rigorous Mie solution for single water droplets showed that important information on average values of both the size and temperature of water droplets can be obtained using the measurements of directional-hemispherical reflectance of submillimeter radiation from the mist layer.

\section{Spectral properties of water droplets}

The spectral optical constants, $n$ and $\kappa$, of pure water are well known [18, 19]. For convenience of subsequent analysis, spectral dependences of these quantities in the most important part of the infrared range are presented in Fig. 1. The spectral characteristics of absorption and scattering of spherical water droplets can be calculated using the Mie theory [2022]. Because of a simplified radiative transfer model used in the present paper, we will focus on two dimensionless far-field characteristics which can be obtained from the analytical Mie solution: the efficiency factor of absorption, $Q_{\mathrm{a}}$, and the transport efficiency factor of scatter- 
ing, $Q_{\mathrm{s}}^{\mathrm{tr}}$. According to Mie theory, the values of $Q_{\mathrm{a}}$ and $Q_{\mathrm{s}}^{\mathrm{tr}}$ depend on both the complex index of refraction $m=n-\mathrm{i} \kappa$ and the diffraction (size) parameter $x=2 \pi a / \lambda$, where $a$ is the droplet radius. The exact Mie calculations are time-consuming especially for large droplets with $x>>1$. Fortunately, one can use the following analytical approximation suggested in paper [23] for semi-transparent particles (see also [24]):

$$
\begin{aligned}
& Q_{\mathrm{a}}=\frac{4 n}{(n+1)^{2}}[1-\exp (-4 \kappa x)] \\
& Q_{\mathrm{s}}^{\mathrm{tr}}=C\left\{\begin{array}{c}
\rho / 5 \text { when } \rho \leq 5 \\
(5 / \rho)^{\gamma} \text { when } \rho>5
\end{array}\right.
\end{aligned}
$$

where

$$
C=1.5 n(n-1) \exp (-15 \kappa) \quad \gamma=1.4-\exp (-80 \kappa)
$$

A comparison of approximation (1a)-(1c) with Mie theory calculations for water droplets at two typical wavelengths is given in Fig. 2. As one can expect, approximate relations give sufficiently accurate results both for $Q_{\mathrm{a}}$ and $Q_{\mathrm{s}}^{\mathrm{tr}}$ in the spectral range of a weak absorption. As to water absorption band (at wavelength $\lambda=3 \mu \mathrm{m}$ ), only the approximation for the absorption efficiency factor is important because $Q_{\mathrm{s}}^{\mathrm{tr}}<<Q_{\mathrm{a}}$ in this spectral range.

The real mists contain water droplets of very different size in every small volume of the mist. Therefore, the calculations of both the absorption coefficient and transport scattering coefficient of the mist at every wavelength should take into account the local size distribution of water droplets. This can be done using the following relations for absorption coefficient and transport scattering coefficient of the mist $[21,24]$ (hereafter the subscript $\lambda$ is omitted for brevity):

$$
\left\{\alpha, \sigma_{\text {tr }}\right\}=0.75 \frac{f_{\mathrm{v}}}{a_{30}} \int_{0}^{\infty}\left\{Q_{\mathrm{a}}, Q_{\mathrm{s}}^{\mathrm{tr}}\right\} a^{2} F(a) d a
$$

where $F(a)$ is the size distribution function. It is convenient to use the following traditional notation:

$$
a_{\mathrm{ij}}=\int_{0}^{\infty} a^{i} F(a) d a / \int_{0}^{\infty} a^{j} F(a) d a
$$

In the case of $i=j+1$, the values of $a_{\mathrm{ij}}$ are the average radii of droplets.

The integration according to Eq. (2) would strongly increase the computational time. Fortunately, the so-called monodisperse approximation when all the particles are assumed to have the same Sauter radius, $a_{32}$, is often applicable $[24,25]$ : 


$$
\alpha=0.75 \frac{f_{\mathrm{v}}}{a_{32}} Q_{\mathrm{a}} \quad \sigma_{\mathrm{tr}}=0.75 \frac{f_{\mathrm{v}}}{a_{32}} Q_{\mathrm{s}}^{\mathrm{tr}}
$$

It was shown in [24] that this simplification may lead to considerable errors in the case when particles of different size have different velocities and/or temperatures. Of course, more detailed calculations should be made to estimate these errors in the problem under consideration. It should be recalled that monodisperse approximation is inapplicable for thermal radiation calculation in the case of a strong difference in temperatures of particles of different size. The known examples are: the thermal radiation of particles in plasma spraying [26, 27], the radiation from two-phase combustion products in exhaust plumes of aluminized-propellant rocket engines [28], and the radiative cooling of core melt droplets in nuclear fuel-coolant interaction $[29,30]$. Nevertheless, the monodisperse approximation is used in the present paper to simplify the mathematical procedure. A verification of this assumption might be a subject of a separate study.

It should be noted that the above consideration is based on the widely used hypothesis of independent scattering [31]. It means that each droplet is assumed to absorb and scatter the radiation in exactly the same manner as if other droplets did not exist. In addition, there is no systematic phase relation between partial waves scattered by individual droplets during the observation time interval, so that the intensities of the partial waves can be added without regard to phase. In other words, each particle is in the far-field zones of all other particles, and scattering by individual particles is incoherent.

\section{Radiative transfer model}

To choose relatively simple but physically sound radiative transfer model, consider the main characteristics of the real problem. First of all, the optical thickness of the mist layer should not be too small to reach a significant attenuation of the incident flame radiation. Therefore, the problem under consideration is characterized by multiple scattering at least in the range of water semi-transparency where the scattering cannot be neglected. In the case of multiple scattering, the details of scattering phase function are not important and one can use the simplest transport approximation [24, 32].

It would be also good to use a set of local 1-D problems instead of considerably more complicated 2-D radiative transfer problem. The use of 1-D solutions is not only the obvious way to simplify the mathematics. It is important that 1-D problems can be solved with sufficiently accuracy using simple differential approximation without any additional iteration. 
Note that a similar approach based on a set of 1-D solutions in the case of relatively small 2-D effects has been recently used in radiative transfer calculations [33].

The schematic presentation of the problem in Fig. 3 makes clear some other assumptions of the computational model: (1) The mist of water droplets is generated by a set of small nozzles at the top of the mist layer; (2) The flat mist layer of constant thickness is considered in the model; (3) One surface of the mist layer is diffusely irradiated by the flame which is also flat but a variation of radiative flux with the height is included in the model.

It is assumed that a protected wall exposed by the transmitted radiation is relatively cold and reflection of the radiation from the wall is negligible. An approximate radiative transfer model used in the present paper is based on a set of 1-D problems for several horizontal layers of the mist curtain. It is assumed that all the mist parameters in every layer are constant and there is no radiative transfer between the neighboring layers (in $z$-direction). As a result, the radiation model is the $z$-direction is similar to the Large-Cell Model suggested for multiphase flows in paper [29]. Of course, the polarization effects due to scattering of radiation by water droplets are negligible, and one can use the scalar radiative transfer theory. With the use of transport approximation, the 1-D radiative transfer equation (RTE) across the mist layer can be written as follows [24, 34, 35]:

$$
\mu \frac{\partial I}{\partial y}+\beta_{\mathrm{tr}} I=\frac{\sigma_{\mathrm{tr}}}{2} \int_{-1}^{1} I(y, \mu) d \mu \quad \mu=\cos \theta \quad 0<y<d
$$

where $I(y, \mu)$ is the spectral radiation intensity at point $y$ in direction $\mu$ (after the integration over the azimuth angles), $\beta_{\mathrm{tr}}=\alpha+\sigma_{\mathrm{tr}}$ is the transport extinction coefficient. The boundary conditions at two surfaces of the mist layer are written as follows:

$$
I(0, \mu)=2 \pi \varepsilon_{\mathrm{f}} I_{\mathrm{b}}\left(T_{\mathrm{f}}\right) \quad I(d,-\mu)=0 \quad \mu>0
$$

where $I_{\mathrm{b}}$ is the Planck function. The above boundary condition at the irradiated surface of the mist denotes that we use the simplest assumption of an optically gray fire radiation in the model problem. In other words, the external spectral radiative flux is assumed to be directly proportional to the blackbody radiation at temperature $T_{\mathrm{f}}$. The coefficient $\varepsilon_{\mathrm{f}}$ is the conventional constant hemispherical emissivity of the flame. It means that integral radiative flux from unit surface area of the flame is expressed as follows:

$$
q_{\mathrm{f}}=\varepsilon_{\mathrm{f}} \int_{0}^{\infty} \pi I_{\mathrm{b}}\left(T_{\mathrm{f}}\right) d \lambda=\varepsilon_{\mathrm{f}} \sigma_{0} T_{\mathrm{f}}^{4}
$$


where $\sigma_{0}$ is the Stefan-Boltzmann constant. This approach is often used in engineering calculations of fire radiation [36].

The diffuse irradiation of the mist makes the problem under consideration much simpler than the problem of shielding of solar radiation by water mist considered in paper [37] because there is no need in a separate consideration of directed and diffuse radiation and the two-flux method can be employed immediately (not only to the diffuse component of the radiation field). According to the two-flux method (Schuster-Schwarzschild approximation [35]) the radiation intensity is presented as a combination of two angular independent components in backward and forward hemispheres:

$$
I(y, \mu)=2 \pi \varepsilon_{\mathrm{f}} I_{\mathrm{b}}\left(T_{\mathrm{f}}\right) \cdot\left\{\begin{array}{l}
J^{-}(y), \mu<0 \\
J^{+}(y), \mu>0
\end{array}\right.
$$

After integration of the RTE over two hemispheres, one can obtain the following boundaryvalue problem for the dimensionless function $g=J^{-}+J^{+}[24]$ :

$$
\begin{gathered}
-\frac{d}{d y}\left(D \frac{d g}{d y}\right)+\alpha g=0 \quad D=1 /\left(4 \beta_{\text {tr }}\right) \\
y=0, \quad D \frac{d g}{d y}=(g-2) / 2 \quad y=d, \quad D \frac{d g}{d y}=-g / 2
\end{gathered}
$$

where $D=1 /\left(4 \beta_{\text {tr }}\right)$ is the spectral radiation diffusion coefficient. The dimensionless spectral radiative flux from the shadow side of the mist layer is

$$
\bar{q}=\frac{q(d)}{2 \pi \varepsilon_{\mathrm{f}} I_{\mathrm{b}}\left(T_{\mathrm{f}}\right)}=\frac{g(d)}{2}
$$

The normalized profile of integral (over the spectrum) radiation power absorbed in the mist is determined as follows:

$$
\bar{W}(y)=\int_{0}^{\infty} \bar{w}(y) d \lambda \quad \bar{w}(y)=\alpha(y) g(y)
$$

Note that the value of $\bar{W}$ is expressed in $\mathrm{m}^{-1}$. It should be recalled that $\bar{w}$ is the spectral value.

It is convenient to introduce dimensionless optical thickness of mist layer measured from the irradiated surface:

$$
\tau_{\mathrm{tr}}(y)=\int_{0}^{z} \beta_{\mathrm{tr}} d y \quad \tau_{\mathrm{tr}, 0}=\tau_{\mathrm{tr}}(d)
$$


In dimensionless variables, equations (9a) and (9b) can be written as follows:

$$
\begin{gathered}
\frac{d^{2} g}{d \tau_{\mathrm{tr}}^{2}}-\xi^{2} g=0 \quad \xi=2 \sqrt{1-\omega_{\mathrm{tr}}} \quad \omega_{\mathrm{tr}}=\sigma_{\mathrm{tr}} / \beta_{\mathrm{tr}} \\
\tau_{\mathrm{tr}}=0, \frac{d g}{d \tau_{\mathrm{tr}}}=2(g-2) \quad \tau_{\mathrm{tr}}=\tau_{\mathrm{tr}, 0}, \quad \frac{d g}{d \tau_{\mathrm{tr}}}=-2 g
\end{gathered}
$$

where $\omega_{\mathrm{tr}}=\sigma_{\mathrm{tr}} / \beta_{\mathrm{tr}}$ is the spectral transport albedo of the medium. In the case of $\omega_{t r}$ independent of $\tau_{\text {tr }}$, one can obtain the following analytical solution to the boundary-value problem $(13 \mathrm{a}, \mathrm{b})$ at $\omega_{\mathrm{tr}}<1(\xi \neq 0)$ :

$$
\begin{gathered}
g=\frac{4\left(E_{\mathrm{tr}, 0}^{2} / E_{\mathrm{tr}}-\gamma E_{\mathrm{tr}}\right)}{(2+\xi)\left(E_{\mathrm{tr}, 0}^{2}-\gamma^{2}\right)} \\
\gamma=\frac{2-\xi}{2+\xi} \quad E_{\mathrm{tr}}=\exp \left(\xi \tau_{\mathrm{tr}}\right) \quad E_{\mathrm{tr}, 0}=\exp \left(\xi \tau_{\mathrm{tr}, 0}\right)
\end{gathered}
$$

The analytical expressions for the dimensionless spectral radiative flux at the shadow side of the mist layer and the profile of absorbed radiation power are as follows:

$$
\begin{gathered}
\bar{q}=\frac{2 E_{\mathrm{tr}, 0}(1-\gamma)}{(2+\xi)\left(E_{\mathrm{tr}, 0}^{2}-\gamma^{2}\right)} \\
W(z)=2 \pi \varepsilon_{\mathrm{f}} \int_{0}^{\infty} \bar{w}(z) I_{\mathrm{b}}\left(T_{\mathrm{f}}\right) d \lambda \quad \bar{w}=\frac{4 \alpha\left(E_{\mathrm{tr}, 0}^{2} / E_{\mathrm{tr}}-\gamma E_{\mathrm{tr}}\right)}{(2+\xi)\left(E_{\mathrm{tr}, 0}^{2}-\gamma^{2}\right)}
\end{gathered}
$$

In real situations, the transport albedo may be not constant across the mist layer and one need a numerical solution to the boundary-value problem (9a)-(9b). Of course, it is not difficult and can be easily done. Nevertheless, it is interesting to consider the simplest case of $\omega_{\mathrm{tr}}=$ const .

To illustrate the above derived analytical solution, consider a case study for the uniform layer of water mist with the average Sauter radius of droplets from $a_{32}=3 \mu \mathrm{m}$ to $a_{32}=30 \mu \mathrm{m}$ at various values of an "attenuation parameter", $A$, of the mist layer. The latter dimensionless parameter is defined as follows:

$$
A=f_{\mathrm{v}} \frac{d}{a_{32}}
$$

The following characteristics of the flame radiation are considered:

$$
T_{\mathrm{f}}=1500 \mathrm{~K} \quad \varepsilon_{\mathrm{f}}=0.9
$$


Considering first the calculated values of $\omega_{\text {tr }}$ in the most important spectral range one can see in Fig. 4 that water mist containing droplets with radius $a_{32}<20 \mu \mathrm{m}$ is a weakly-scattering medium only in the wavelength range of $2.7<\lambda<3.2 \mu \mathrm{m}$, whereas the scattering is considerable or even predominant outside this range. As to the short-wave range of $\lambda<2.5 \mu \mathrm{m}$, the scattering cannot be ignored even in the case of $a_{32}=50 \mu \mathrm{m}$ (see Fig. $4 \mathrm{~b}$ ). It is also confirmed by the data of Figs. $4 a$ and $4 b$ that analytical relations (1a)-(1c) give an acceptable approach which can be used instead of time-consuming Mie theory calculations. Therefore, these approximate relations are used in subsequent calculations.

The main results of radiative transfer calculations are presented in Fig. 5. The integral (over the spectrum) transmitted radiative flux is determined as follows.

$$
q_{\mathrm{t}}=2 \pi \varepsilon_{\mathrm{f}} \int_{0}^{\infty} \bar{q} I_{\mathrm{b}}\left(T_{\mathrm{f}}\right) d \lambda
$$

According to Fig. 5, the value of $q_{\mathrm{t}}$ decreases strongly with the effective thickness of water mist layer. The curves of $q_{\mathrm{t}}(A)$ in the important range of $3<a_{32}<30 \mu \mathrm{m}$ are similar to each other and the value of $q_{\mathrm{t}}$ at a fixed value of $A$ decreases slightly with the droplet size. So, the attenuation parameter $A$ is the most important quantity which determines the radiative flux transmitted through the mist layer.

It is also interesting to determine the radiative flux reflected from the irradiated surface of the mist layer. The dimensionless reflectance can be obtained as follows:

$$
R_{\mathrm{h}}=g(0)-1
$$

With the use of analytical solution (14a) we have:

$$
R_{\mathrm{h}}=\frac{4}{2+\xi} \frac{1-\gamma / E_{\mathrm{tr}, 0}^{2}}{1-\gamma^{2} / E_{\mathrm{tr} .0}^{2}}-1
$$

In the limiting case of purely absorbing (non-scattering) medium ( $\xi=2$ and $\gamma=0$ ) this leads to the physically obvious result of $R_{\mathrm{h}}=0$. The opposite case of a scattering but non-absorbing medium ( $\xi=0$ ) cannot be considered using the above solution because of obvious degeneration of the equation (13a) [24]. At the same time, one can use Eq. (21) to obtain the following important relation for the limit of optically thick mist layer:

$$
R_{\mathrm{h}}=\frac{1-\sqrt{1-\omega_{\mathrm{tr}}}}{1+\sqrt{1-\omega_{\mathrm{tr}}}}
$$


Equation (22) describes correctly the increase of radiation reflection with the medium transport albedo and can be used in engineering estimates.

Considering now a variation of the total radiation power, $W_{\text {tot }}$, absorbed in the mist:

$$
W_{\mathrm{tot}}=2 \pi \varepsilon_{\mathrm{f}} \int_{0}^{\infty} \bar{w}_{\mathrm{tot}} I_{\mathrm{b}}\left(T_{\mathrm{f}}\right) d \lambda
$$

This quantity is important because there is an obvious relation between the value of $W_{\text {tot }}$ and the required flow rate of water. One can use the following radiative balance equation for the spectral values:

$$
\bar{w}_{\mathrm{tot}}=0.5\left(1-R_{\mathrm{h}}\right)-\bar{q}
$$

Having substituted Eqs. (15) and (21), the following analytical relation can be obtained:

$$
\bar{w}_{\mathrm{tot}}=1-\frac{2}{2+\xi} \frac{1+(1-\gamma) / E_{\mathrm{tr}, 0}-\gamma / E_{\mathrm{tr}, 0}^{2}}{1-\gamma^{2} / E_{\mathrm{tr} .0}^{2}}
$$

The results of calculations with the use of analytical solution (25) are presented in Fig. 6. One can see that the absorbed radiation power $W_{\text {tot }}$ increases monotonically with both the attenuation parameter and the droplet size but the effect of these parameters is relatively insignificant in the ranges of $A>3$ and $a_{32}>20 \mu \mathrm{m}$. This is an important qualitative result which should be further examined on the basis of more detailed analysis of real water mists.

It should be noted that the formal use of the results obtained in the above radiative transfer analysis is insufficient to chose a shielding mist to protect an object wall from the intense fire radiation. The mist containing relatively small droplets looks more promising because of great value of the attenuation parameter but the volumetric absorption of the incident radiation near the irradiated surface of the mist and small velocities of the falling droplets will lead to high rate of the mist evaporation. In the opposite case of very large droplets, the radiation is not practically reflected from the mist because of low scattering and also a considerable attenuation of the fire radiation can be reached only in the case of a geometrically thick mist layer with high flow rate of water. Of course, the latter variant is not the optimal one. Most likely, the droplets of an average size may be a good choice.

One should recall that thermal conditions near the irradiated surface of the mist and the conditions at the opposite shadow side of the mist are quite different. This makes interesting the use of more sophisticated engineering solution with a variable size of supplied water droplets across the mist layer. It is natural to have relatively large droplets with average radius $a_{32}^{(1)}$ 
at the irradiated side and much smaller droplets with radius $a_{32}^{(2)}<<a_{32}^{(1)}$ at the shadow side of the mist layer. This idea will be examined on the basis of the case study considered below.

\section{Transient heat transfer model}

Strictly speaking, the heat transfer model for water mist exposed by thermal radiation from fire should be based on CFD modeling of the flow field and convective heat transfer in combination with radiative heat transfer modeling. The general problem is too complicated especially because of possible dynamic and thermal non-equilibrium of evaporating water droplets. On the other hand, the practical sense of detailed modeling is not obvious at the moment because of great uncertainty in many parameters of particular processes. In the present paper, a simplified problem statement is considered without some details which can be ignored at this stage of the research.

First of all, it is assumed that the shape of the main stream region can be presented as a plane-parallel layer (see Fig. 3) and the effects of viscous boundary interaction with ambient air can be ignored. Following the above principal suggestion, we consider the mist containing two separate layers. It is assumed that the first layer (from the flame side) contains large droplets and the second layer contains relatively small droplets. From the engineering point of view, such approach will provide more stability to the curtain as the larger droplets have higher momentum where as the smaller droplets will achieve higher attenuation.

Generally speaking, the gas flow with suspended droplets is characterized by dynamic and thermal nonequilibrium of water droplets with respect to the gas flow. But this effect is degenerated in the limits of both large and small droplets. In the first case, the decrease in droplet velocity due to the drag force is relatively small, whereas the motion of small droplets is determined by the gas flow.

The specific effects on parameters of small water droplets in the entrance region of the flow characterized by and also the hydrodynamic effects in the vicinity of the ground surface that is impinged by a mist flow are not considered. This is a natural assumption because the region of the mist formation may be positioned at a greater height that the fire and the part of fire characterized by a significant thermal radiation is usually observed at some distance from the ground. In other words, we consider a middle part of the long mist layer.

The following modes of heat transfer seem to be the most important and should be included in the computational model: the heating of water droplets by external radiation from fire, partial evaporation of these droplets, and the falling flow of the mist. One cannot exclude that 
turbulent heat transfer across the mist layer should be also taken into account, but the liming volume of journal paper does not allow to analyze this effect.

The radiative heating of water droplets can be calculated using the field of radiation power absorbed in the mist. This part of the general model was a subject of previous sections of the paper. The droplet size is not constant across the composite mist layer and also because of evaporation under the action of nonuniform absorption of fire radiation. As a result, the medium transport albedo is also not constant and one should use numerical solution to the boundary-value problem (13a)-(13b) instead of analytical solution (14a)-(14b).

It is assumed that water droplets are isothermal and their temperature is the same as that of ambient gas. It means that radiation power is spent to heat both droplets and gas and also to evaporate the droplets. The simplest equilibrium evaporation model is considered and the evaporation at temperatures less than the saturation temperature at normal atmospheric conditions, $T_{\mathrm{s}}=373 \mathrm{~K}$, is neglected. Possible overheating of water droplets is also not considered in the model. The effects of surface tension are neglected.

The nonuniform volumetric heating of large water droplets and more accurate analysis of droplet evaporation in presence of thermal radiation are not considered in the present paper. The details of more comprehensive models can be found in the literature [38-42]. Note that the monodisperse approximation is employed for both layers of the mist and the evaporation is treated as the only effect resulting in change of the droplet size. In other words, possible effects of agglomeration or fragmentation of droplets are not considered.

The falling flow of the mist is not calculated in the paper and effects of steam generation and lift forces due to the gas heating are not taken into account. Instead, two parallel uniform flows with constant velocities are considered. Of course, this is a strong assumption, but this approach seems to be an important stage of the general study for possible gradual size variation of supplied droplets on the basis of much more detailed CFD calculations.

An applicability of the assumption of local dynamic equilibrium of small water droplets and ambient air flow can be analyzed on the basis of the following equation for the motion of a spherical water droplet in viscous gas in $z$-direction (see Fig. 3) [43]:

$$
\frac{4}{3} \pi a^{3} \rho_{\mathrm{w}} \frac{d u_{\mathrm{d}}}{d t}=\frac{1}{2} C_{\mathrm{D}} \pi a^{2} \rho\left(u-u_{\mathrm{d}}\right)^{2}
$$

where $u$ and $u_{\mathrm{d}}$ are the gas and droplet velocities, $\rho$ and $\rho_{\mathrm{w}}$ are the densities of gas and water, $C_{\mathrm{D}}$ is the drag coefficient. For slow relative motion (when Reynolds number $\operatorname{Re}_{\mathrm{d}}=2 \rho\left|u-u_{d}\right| a / \eta<<1$, the case of small particles - the Stokes flow) the drag coefficient is as follows: 


$$
C_{D}=24 / \operatorname{Re}_{d}
$$

and Eq. (26) can be written in the form:

$$
t_{\text {rel }} \frac{d u_{\mathrm{d}}}{d t}=u-u_{d} \quad t_{\text {rel }}=\frac{2}{9} \frac{\rho_{\mathrm{w}} a^{2}}{\eta}
$$

where $t_{\text {rel }}$ is the relaxation time. Note that it is sometimes convenient to introduce the Stokes number defined as Stk $=t_{\text {rel }}|\nabla \vec{u}|$ to estimate the dynamic nonequilibrium of inertial particles in a viscous medium $[43,44]$. In our case, it is sufficient to compare directly the length of the droplet relaxation path and the height of a single horizontal layer of the computational region (see Fig. 3). According to [9], the following value of an average initial velocity of water droplets is used in the estimates:

$$
u_{\mathrm{d}, 0}=5 \mathrm{~m} \mathrm{~s}^{-1}
$$

The calculations for water droplets with radius of $a=30 \mu \mathrm{m}$ showed that $u_{\mathrm{d}, 0} t_{\text {rel }} \approx 54 \mathrm{~mm}<<\Delta H=H / N$ at $H=10 \mathrm{~m}$ and $N \leq 100$. It means that dynamic nonequilibrium of small water droplets can be neglected in the simplified computational model. It assumed also that there is a thermal equilibrium between the droplets and ambient gas in every horizontal layer of small droplets. This approach is not good for the upper layer but it seems not so important for the simplified model problem. As a result, the only local temperature is used at every point of the second layer of the mist. Moreover, it is assumed that the gas medium with suspended particles can be treated as an equivalent continuous medium with average local dynamic and thermal parameters. The initial velocity of the medium in this mist layer is determined as follows:

$$
u_{2} \approx u_{\mathrm{d}, 0} f_{\mathrm{v}, \text { in }} \rho_{\mathrm{w}} / \rho_{\mathrm{g}}
$$

On the contrary, it is assumed that velocity of large water droplets in the first layer of the mist is constant and the presence of ambient gas has no effect on the droplet motion. As to variation of the droplet temperature from the initial value to the saturation temperature, it is determined by the local volumetric absorption of the fire radiation.

The computational model is based on dividing the mist volume into several horizontal layers for subsequent solution of a set of coupled 1-D heat transfer problems for every layer of the same thickness starting from the upper layer $j=1$ (see Fig. 3). The number of layers, $N$, was varied in the methodological calculations to reach an acceptable computational error.

In the case of negligible turbulent heat transfer across the mist layer, the approximate mathematical formulation of a heat transfer problem for every layer is as follows: 


$$
(\rho c)_{\mathrm{j}} u \frac{T_{\mathrm{j}+1}^{*}(y)-T_{\mathrm{j}}(y)}{\Delta H}=W_{\text {rad }, \mathrm{j}}(y) \quad T_{1}(y)=T_{0} \quad j=1, \ldots, N-1
$$

where

$$
\begin{gathered}
u=u_{1} \text { and }(\rho c)_{\mathrm{j}}=f_{\mathrm{v}, \mathrm{j}}(\rho c)_{\mathrm{w}} \text { at } 0<y<y_{*} \\
u=u_{2} \text { and }(\rho c)_{\mathrm{j}} \approx(\rho c)_{\mathrm{g}}+f_{\mathrm{v}, \mathrm{j}}(y)(\rho c)_{w} \text { at } y_{*}<y<d
\end{gathered}
$$

It is assumed here that $f_{\mathrm{v}, \mathrm{j}}<<1$ and variation of volumetric heat capacity of gas mixture due to evaporation of water is insignificant.

Equation (31a) can be considered as a result of the use of an explicit finite-difference scheme for the obvious differential equation. In the case of $T_{\mathrm{j}+1}^{*}(y) \leq T_{\mathrm{s}}$, where $T_{\mathrm{s}}=373 \mathrm{~K}$ is the saturation temperature, the value of $T_{\mathrm{j}+1}^{*}$ is the real temperature and $T_{\mathrm{j}+1}(y)=T_{\mathrm{j}+1}^{*}(y)$. When the formal use of Eq. (30a) gives $T_{\mathrm{j}+1}^{*}(y)>T_{\mathrm{s}}$, the saturation temperature is reached: $T_{\mathrm{j}+1}(y)=T_{\mathrm{s}}$. As to the current volume fraction of water droplets, it can be estimated using the following relation:

$$
f_{v, \mathrm{j}+1}(y)=f_{v, \mathrm{j}}(y)\left[1-\rho_{\mathrm{g}} \frac{W_{\mathrm{rad}, \mathrm{j}}(y)}{L} \frac{\Delta H}{u} \frac{T_{\mathrm{j}+1}^{*}-T_{\mathrm{s}}}{T_{\mathrm{j}+1}^{*}-T_{j}}\right]
$$

Obviously, $W_{\text {rad } \mathrm{j}}(y)=0$ at $f_{v, \mathrm{j}}(y)=0$ and negative values of $f_{v, \mathrm{j}+1}(y)$ should not appear in correct calculations.

According to the traditional classification of combined heat transfer problems [45], the above problem is an example of the so-called radiative heat transfer in moving media. The radiative boundary layer (an outer part of the thermal boundary layer in the case of optically thin viscous boundary layer) and the liquid droplet radiator for space applications are two known examples of the same class of heat transfer problems [21, 24]. In our particular case, the problem is a bit more complicated because of two-layered medium and evaporation of water droplets.

\section{Results for the case study}

The following values of input parameters were used in the case study: $H=10 \mathrm{~m}, d=1 \mathrm{~m}$, $T_{0}=300 \mathrm{~K}, \rho_{\mathrm{w}}=10^{3} \mathrm{~kg} \mathrm{~m}^{-3}, \rho_{\mathrm{g}}=1 \mathrm{~kg} \mathrm{~m}^{-3}, \quad f_{\mathrm{v}, 1}=f_{\mathrm{v}, \mathrm{in}}=10^{-4}, \quad a_{32}^{(1)}=100 \mu \mathrm{m}, \quad a_{32}^{(2)}=30 \mu \mathrm{m}$, $u_{1}=u_{\mathrm{d}, 0}=3 \mathrm{~m} \mathrm{~s}^{-1}, c_{\mathrm{w}}=4.18 \mathrm{~kJ} \mathrm{~kg}^{-1} \mathrm{~K}^{-1}, c_{\mathrm{g}}=1 \mathrm{~kJ} \mathrm{~kg}^{-1} \mathrm{~K}^{-1}, L=2.26 \mathrm{MJ} \mathrm{kg}^{-1}$. Three variants of thickness of the front (irradiated) vertical layer of the mist are considered: $y_{*}=0,0.2 \mathrm{~m}$, and $0.5 \mathrm{~m}$. The first of these variants corresponds to the uniform mist containing only small water 
droplets, two other variants illustrate the effect of large droplets supplied from the side of fire. Note that numerical integration over the spectrum was conducted for the wavelength interval from $\lambda_{1}=0.3 \mu \mathrm{m}$ to $\lambda_{2}=6 \mu \mathrm{m}$. The uniform step of $\Delta \lambda=0.02 \mu \mathrm{m}$ was used in the spectral integration.

Some results of calculations for the case study are presented in Figs. 7 and 8. One can see in Fig. 7 that the presence of large droplets at the irradiated side of the mist layer may decrease the transmitted radiative flux to the lower part of the protected object. This is explained by more favorable profile of the droplet size and the resulting volume fraction of water droplets (see Fig. 8). This particular result confirms potential advantages of the suggested decrease of the size supplied water droplets with the distance from the irradiated surface of the mist layer.

\section{Possible microwave monitoring of water mist parameters}

Microwave absorption and scattering by water droplets have been studied during many years because of developing applications in remote sensing of the ocean ant atmosphere [4648]. In this paper, possible microwave monitoring of the main parameters of a water mist is considered because this technique might be an important part of the mist shielding properties. To analyze a possibility of such monitoring, consider first the microwave optical constants of water in the millimeter and centimeter spectral ranges. Following [49], the complex dielectric constant of water is determined by the Debye relaxation model and the following modified equations:

$$
\begin{aligned}
& \varepsilon^{\prime}=\varepsilon_{\infty}+\frac{\left(\varepsilon_{\mathrm{s}}-\varepsilon_{\infty}\right)\left(1+\left(\bar{\lambda}_{\mathrm{s}}\right)^{1-\varphi} S\right)}{1+2\left(\bar{\lambda}_{\mathrm{s}}\right)^{1-\varphi} S+\left(\bar{\lambda}_{\mathrm{s}}\right)^{2-\varphi}} \\
& \varepsilon^{\prime \prime}=\frac{\varepsilon_{\mathrm{s}}-\varepsilon_{\infty}}{1+2\left(\bar{\lambda}_{\mathrm{s}}\right)^{1-\varphi} S+\left(\overline{\lambda_{\mathrm{s}}}\right)^{2-\varphi}}\left(\bar{\lambda}_{\mathrm{s}}\right)^{1-\varphi} C+\frac{\sigma_{\mathrm{el}} \lambda}{18.8496} 10^{-11} \\
& \bar{\lambda}_{\mathrm{s}}=\lambda_{\mathrm{s}} / \lambda \quad S=\sin (\varphi \pi / 2) \quad C=\cos (\varphi \pi / 2)
\end{aligned}
$$

where $\lambda_{\mathrm{s}}$ is the relaxation wavelength, $\varepsilon_{\infty}$ and $\varepsilon_{\mathrm{s}}$ are the high- and low-frequency dielectric constants. Hereafter, the values of $\lambda$ and $\lambda_{\mathrm{s}}$ are expressed in millimeters. Equations (33a)(33c) are reduced to the classical Debye equations when the electrical conductivity $\sigma_{\mathrm{el}}$ and the spread parameter $\varphi$ are equal to zero. In calculations for water, the constant value of $\sigma_{\mathrm{el}}=12.5664 \cdot 10^{8}(\mathrm{Ohm} \mathrm{mm})^{-1}$ was used and other parameters were obtained using the following relations: 


$$
\begin{gathered}
\varepsilon_{\infty}=5.27137+0.0216474 T-0.00131198 T^{2} \\
\varphi=-16.8129 /(T+273)+0.0609265 \\
\lambda_{\mathrm{s}}=0.00033836 \exp [2513.98 /(T+273)] \\
\varepsilon_{\mathrm{s}}=78.54\left(1-4.579 \cdot 10^{-3} \tilde{T}+1.19 \cdot 10^{-5} \tilde{T}^{2}-2.8 \cdot 10^{-8} \tilde{T}^{3}\right) \quad \tilde{T}=T-25
\end{gathered}
$$

Note that temperature $T$ in the above equations is expressed in centigrade degrees.

The calculated spectral dependences of refraction and absorption indices of water in the most promising parts of sub-millimeter and millimeter spectral ranges are presented in Fig 9. The index of refraction of water increases both with wavelength and temperature. The latter is true at wavelength $\lambda>0.85 \mathrm{~mm}$. The spectral and temperature dependences of absorption index in a long-wave part of the considered spectral range are more complex (Fig. 9b). It is clear that one cannot expect a significant absorption of radiation by water droplets at $\lambda>2 \mathrm{~mm}$ where the index of refraction is too large [24]. At the same time, a combination of moderate values of $n$ and fast increasing values of $\kappa$ in the sub-millimeter wavelength range will result in unusual optical properties of single water droplets.

Consider the optical properties of small water droplets typical of water mist. As above, we assume that droplets are spherical, their volume fraction is small and they are randomly positioned in space. The last assumptions are important but insufficient to neglect the so-called near-field dependent scattering effects. One should recall the effect of coherent microwave scattering by clusters of water droplets is sometimes observed in cumulus clouds in turbulent atmosphere [50-52]. We assume that there is no such an effect in the problem under consideration and the hypothesis of independent scattering by single water droplets of mists is true also in the microwave spectral range.

Some results of calculations for single droplets are presented in Figs. 10 and 11. The limiting volume of the paper makes possible to illustrate only the most interesting effects observed in sub-millimeter spectral range. The strong resonances of absorption like those studied in detail for gold nanoparticles in the so-called therapeutic window $(0.6<\lambda<1.4 \mu \mathrm{m})$ are observed. These nanoparticles are widely used in hyperthermia of tumors and other medical applications related with targeted heating of biological tissues [53-55]. But it is not the same effect because of multiple resonances and the accompanying resonances of scattering. The effect of water temperature appears to be also significant. Note that similar data for resonance absorption of sub-millimeter radiation by single water droplets have been recently reported in [56]. The computational data for the transport scattering albedo presented in Fig. 11 are especially important because they indicate possible monitoring of the main parameters of water 
mists. Of course, it is a separate problem which is beyond the scope of the present paper. Therefore, we limit our consideration by computational results for the normal-hemispherical reflectance of a uniform optically thick mist [57]:

$$
R_{\mathrm{n}-\mathrm{h}}=\frac{\omega_{t r}}{\left(1+\sqrt{1-\omega_{t r}}\right)\left(1+2 \sqrt{1-\omega_{t r}}\right)}
$$

Figure 12a indicates that the measurements of $R_{\mathrm{n}-\mathrm{h}}$ at wavelength $\lambda=0.5 \mathrm{~mm}$ can be used to estimate an average droplet temperature in the case of $10<a<25 \mu \mathrm{m}$ without more detailed information on size of water droplets. As to the average droplet size, it can be obtained in the case of $40<a<50 \mu \mathrm{m}$ from the measurements at wavelength $\lambda=0.7 \mathrm{~mm}$, and the result will be independent of droplet temperature (see Fig. 12b). Note that possible measurements of reflectance from the shadow side of the mist layer will give us average values of both the droplet size and temperature in this relatively cold and not evaporated region of the mist. More detailed information on variation of the retrieved values with the distance from the shadow surface of the mist layer can be obtained from the measurements of directional-hemispherical reflectance at various angles of incidence.

\section{Conclusions}

A simplified theoretical model for attenuation of fire radiation by water mist was developed. This spectral model is based on calculated absorption and scattering characteristics of water droplets, the local 1-D solutions for radiative heat transfer through the mist layer, and transient heat transfer model taking into account heating and evaporation of the droplets.

The computational data illustrate the main special features of the problem and enable one to estimate the effects of an average droplet size, the volume fraction of water, and the mist layer thickness on quality of the fire protection. The suggested analytical solution for radiative transfer and also the results of numerical analysis of heat transfer in the mist are expected to be useful for physically sound engineering estimates.

The case study for the fire radiation protection by water mist is considered. It was suggested to decrease the size of supplied water droplets with the distance from the irradiated surface of the mist layer. The results of numerical calculations confirmed potential advantages of this engineering solution.

A possibility of microwave monitoring of water mist parameters was also considered in the paper. It was shown that important information on average values of both the size and temperature of water droplets can be obtained using the measurements of directionalhemispherical reflectance of sub-millimeter radiation from the mist layer. 


\section{Conflict of interests}

None declared.

\section{Acknowledgements}

This study was supported by the UK Royal Academy of Engineering (Grant DVF1415/2/22)

and the Russian Foundation for Basic Research (Grants no. 13-08-00022a and 16-08-00157a). 


\section{References}

[1] M.A. Rana, Y. Guo, and M.S. Mannan, Use of water spray curtain to disperse LNG vapor clouds, J. Loss Prevent. Proc. Indust. 23 (1) (2010) 77-88.

[2] Z. Liu and A.K. Kim, A Review of Water Mist Fire Suppression Technology: Part IIApplication Studies, J. of Fire Prot. Eng. 11 (1) (2001) 16-42.

[3] J.-F. Sacadura, Radiative heat transfer in fire safety science, J. Quant. Spectr. Radiat. Transfer 93 (1-3) (2005) 5-24.

[4] S. Stephenson and M.J. Coward, Attenuation of radiant heat on LNG/LPG carriers with freestanding water curtains, Gastech 86 LNG/LPG conference, Hamburg, 1986, pp. $157-168$.

[5] W.Y. Cheung, Radiation blockage of water curtains, Int. J. Eng. Perf.-Based Fire Codes 1 (2009) 7-13.

[6] T.S. Ravigururajan and M.R. Beltran, A model for attenuation of fire radiation through water droplets, Fire Safety J. 15 (2) (1989) 171-181.

[7] A. Coppalle, D. Nedelka, and B. Bauer, Fire protection: Water curtains, Fire Safety J. 20 (1993) 241-255.

[8] S. Dembele, J.X. Wen, and J.-F. Sacadura, Analysis of the two-flux model for predicting water spray transmittance in fire protection application, ASME J. Heat Transfer 122 (1) (2000) 183-186.

[9] J.-M. Buchlin, Thermal shielding by water spray curtain, J. Loss Prevent. Proc. Indust. 18 (4-6) (2005) 423-432.

[10] C.C. Tseng and R. Viskanta, Absorptance and transmittance of water spray/mist curtains, Fire Safety J. 42 (2) (2007) 106-114.

[11] S. Dembele, A. Delmas, and J.-F. Sacadura, A method for modeling the mitigation of hazardous fire thermal radiation by water spray curtains, ASME J. Heat Transfer 119 (4) (1997) 746-753.

[12] N. Berour, D. Lacroix, P. Boulet, and G. Jeandel, Radiative and conductive heat transfer in a nongrey semitransparent medium. Application to fire protection curtains, J. Quant. Spectr. Radiat. Transfer 86 (1) (2004) 9-30.

[13] A. Collin, P. Boulet, D. Lacroix, and G. Jeandel, On radiative transfer in water spray curtains using the discrete ordinates method, J. Quant. Spectr. Radiat. Transfer 92 (1) (2005) 85-110.

[14] P. Boulet, A. Collin, and G. Parent, Heat transfer through a water spray curtain under the effect of a strong radiative source, Fire Safety J. 41 (1) (2006) 15-30. 
[15] S. Hostikka and K. McGrattan, Numerical modeling of radiative heat transfer in water sprays, Fire Safety J. 41 (1) (2006) 76-86.

[16] A. Collin, P. Boulet, G. Parent, and D. Lacroix, Numerical simulation of water spray Radiation attenuation related to spray dynamics, Int. J. Thermal Sci. 46 (9) (2007) 856868.

[17] A. Collin, P. Boulet, G. Parent, M.P. Vetrano, and J.M. Buchlin, Dynamics and thermal behavior of water sprays, Int. J. Thermal Sci. 47 (4) (2008) 399-407.

[18] G.M. Hale and M.P. Querry, Optical constants of water in the $200 \mathrm{~nm}$ to $200 \mu \mathrm{m}$ wavelength region, Appl. Optics 12 (3) (1973) 555-563.

[19] V.M. Zolotarev and A.V. Dyomin, Optical constants of water in wide wavelength range $0.1 \AA \circ \div 1 \mathrm{~m}$, Opt. Spectr. 43 (2) (1977) 271-279.

[20] C.F. Bohren and D.R. Huffman, Absorption and Scattering of Light by Small Particles, Wiley, New York, 1983.

[21] L.A. Dombrovsky, Radiation Heat Transfer in Disperse Systems, Begell House, New York, 1996.

[22] W. Hergert and T. Wriedt, The Mie Theory: Basics and Applications, Springer, Berlin, 2012 .

[23] L.A. Dombrovsky, Spectral model of absorption and scattering of thermal radiation by droplets of diesel fuel, High Temp. 40 (2) (2002) 242-248.

[24] L.A. Dombrovsky and D. Baillis, Thermal Radiation in Disperse Systems: An Engineering Approach, Begell House, New York, 2010.

[25] W.F. Godoy and P.E. Des Jardin, Efficient transmission calculation for polydisperse water sprays using spectral scaling, J. Quant. Spectr. Radiat. Transfer 108 (2007) 440453.

[26] L.A. Dombrovsky and M.B. Ignatiev, An estimate of the temperature of semitransparent oxide particles in thermal spraying, Heat Transfer Eng. 24 (2) (2003) 60-68.

[27] P. Fauchais, G. Montavon, and G. Bertrand, From powders to thermally sprayed coatings, J. Therm. Spray Tech. 19 (1-2) (2010) 56-80.

[28] G. Cai, D. Zhu, and X. Zhang, Numerical simulation of the infrared radiative signatures of liquid and solid rocket plumes, Aerospace Sci. Tech. 11 (6) (2007) 473-480.

[29] L.A. Dombrovsky, Large-cell model of radiation heat transfer in multiphase flows typical for fuel-coolant interaction, Int. J. Heat Mass Transfer 50 (17-18) (2007) 34013410. 
[30] L.A. Dombrovsky, M.V. Davydov, and P. Kudinov, Thermal radiation modeling in numerical simulation of melt-coolant interaction, Comput. Thermal Sci. 1 (1) (2009) 135.

[31] M.I. Mishchenko, Electromagnetic Scattering by Particles and Particle Groups: An Introduction. Cambridge (UK): Cambridge Univ. Press, 2014.

[32] L.A. Dombrovsky, The use of transport approximation and diffusion-based models in radiative transfer calculations, Comput. Therm. Sci. 4 (4) (2012) 297-315.

[33] L.A. Dombrovsky, V. Timchenko, C. Pathak, H. Piazena, W. Müller, and M. Jackson, Radiative heating of superficial human tissues with the use of water-filtered infrared-A radiation: A computational modeling, Int. J. Heat Mass Transfer 85 (2015) 311-320.

[34] J.R. Howell, R. Siegel, and M.P. Mengüç, Thermal Radiation Heat Transfer, CRC Press, New York, 2010.

[35] M.F. Modest, Radiative Heat Transfer, Third edition, Academic Press, New York, 2013.

[36] J.L. de Ris, P.K. Wu, and G. Heskestad, Radiation fire modeling, Proc. Combust. Inst. 28 (2000) 2751-2759.

[37] L.A. Dombrovsky, V.P. Solovjov, and B.W. Webb, Attenuation of solar radiation by water mist and sprays from the ultraviolet to the infrared range, J. Quant. Spectr. Radiat. Transfer 112 (7) (2011) 1182-1190.

[38] L.A. Dombrovsky, Absorption of thermal radiation in large semi-transparent particles at arbitrary illumination of the polydisperse system, Int. J. Heat Mass Transfer 47 (25) (2004) 5511-5522.

[39] S. Sazhin, Advanced models of fuel droplet heating and evaporation, Prog. Energy Combust. Sci. 32 (2) (2006) 162-214.

[40] C.C. Tseng and R. Viskanta, Enhancement of water droplet evaporation by radiation absorption, Fire Safety J. 41 (3) (2006) 236-247.

[41] G. Miliauskas and V. Sabanas, Interaction of transfer processes during unsteady evaporation of water droplets, Int. J. Heat Mass Transfer 49 (11-12) (2006) 1790-1803.

[42] M.Q. Brewster, Evaporation and condensation of water mist/cloud droplets with thermal radiation, Int. J. Heat Mass Transfer 88 (2015) 695-712.

[43] C.T. Crowe, J.D. Schwarzkopf, M. Sommerfeld, and Y. Tsuji, Multiphase Flows with Droplets and Particles, Second edition, CRC Press, New York, 2011.

[44] L.I. Zaichik, V.M. Alipchenkov, and E.G. Sinaiski, Particles in Turbulent Flows, Wiley, Weinheim, 2008.

[45] E.M. Sparrow and R.D. Cess, Radiation Heat Transfer, McGraw-Hill, New York, 1978. 
[46] T. Oguchi, Electromagnetic wave propagation and scattering in rain and other hydrometeors, Proc. IEEE 71 (9) (1983) 1029-1078.

[47] L.A. Dombrovsky and V.Y. Raizer, Microwave model of a two-phase medium at the ocean surface, Izvestiya, Atmos. Ocean. Phys. 28 (8) (1992) 650-656.

[48] V. Cherny and V.Y. Raizer, Passive Microwave Remote Sensing of Oceans, Wiley, New York, 1998.

[49] P.S. Ray, Broadband complex refractive indices of ice and water, Appl. Optics 11 (8) (1972) 1836-1844.

[50] R.A. Shaw, Particle-turbulence interactions in atmospheric clouds, Ann. Rev. Fluid Mech. 35 (2003) 183-227.

[51] L.A. Dombrovsky and L.I. Zaichik, An effect of turbulent clustering on scattering of microwave radiation by small particles in the atmosphere, J. Quant. Spectr. Radiat. Transfer 111 (1) (2010) 234-242.

[52] K. Matsuda, R. Onishi, M. Hirahara, R. Kurose, K. Takahashi, S. Komori, Influence of microscale turbulent droplet clustering on radar cloud observations, J. Atmos. Sci. 71 (2014) 3569-3582.

[53] N.G. Khlebtzov and L.A. Dykman, Optical properties and biomedical applications of plasmonic nanoparticles, J. Quant. Spectr. Radiat. Transfer 111 (1) (2010) 1-35.

[54] L.A. Dombrovsky, V. Timchenko, M. Jackson, and G.H. Yeoh, A combined transient thermal model for laser hyperthermia of tumors with embedded gold nanoshells, Int. J. Heat Mass Transfer 54 (25-26) (2011) 5459-5469.

[55] Y. Bayazitoglu, S. Kheradmand, and T.K. Tullius, An overview of nanoparticle assisted laser therapy, Int. J. Heat Mass Transfer 67 (2013) 469-486.

[56] L.A. Dombrovsky, A.A. Fedorets, and D.N. Medvedev, The use of infrared irradiation to stabilize levitating clusters of water droplets, Infrared Phys. Tech. (2016) in press (accepted on Dec. 21, 2015).

[57] L. Dombrovsky, J. Randrianalisoa, and D. Baillis, Modified two-flux approximation for identification of radiative properties of absorbing and scattering media from directionalhemispherical measurements, J. Opt. Soc. Am. A 23 (1) (2006) 91-98. 


\section{Figure captions}

Fig. 1. Spectral indices of refraction (a) and absorption (b) of pure water [reference?].

Fig. 2. Efficiency factor of absorption and transport efficiency factor of scattering for water droplets of various radius: $1-\lambda=3 \mu \mathrm{m}, 2-\lambda=5 \mu \mathrm{m}$; solid lines - Mie theory, dashed lines - approximation (1a-c).

Fig. 3. Scheme of the problem for diffuse irradiation of water mist layer.

Fig. 4. Transport scattering albedo of monodisperse water mists: 1 - Mie theory, 2 - approximation (1a-c).

Fig. 5. Radiative flux transmitted through the mist layer.

Fig. 6. Integral radiation power absorbed in the mist layer.

Fig. 7. Radiative flux transmitted though the mist layer of height $10 \mathrm{~m}$ at different thickness of the front layer of supplied large water droplets: $1-y_{*}=0$ (no large droplets), $2-$ $y_{*}=0.2 \mathrm{~m}, 3-y_{*}=0.5 \mathrm{~m}$.

Fig. 8. Profiles of Sauter's mean radius of water droplets (a) and local volume fraction of water (b) at the lower cross section of water mist layer: $1-y_{*}=0,2-y_{*}=0.2 \mathrm{~m}, 3-$ $y_{*}=0.5 \mathrm{~m}$.

Fig. 9. Indices of refraction (a) and absorption (b) for water in sub-millimeter and millimeter wavelength ranges: $1-T=20^{\circ} \mathrm{C}, 2-50^{\circ} \mathrm{C}, 3-80^{\circ} \mathrm{C}$.

Fig. 10. Efficiency factor of absorption and transport efficiency factor of scattering for water droplets in the sub-millimeter at wavelengths $0.5 \mathrm{~mm}$ (a) and $0.7 \mathrm{~mm}(\mathrm{~b}): 1-T=20^{\circ} \mathrm{C}$, $2-80^{\circ} \mathrm{C}$.

Fig. 11. Transport albedo of a monodisperse water mist in the sub-millimeter range at wavelengths $0.5 \mathrm{~mm}$ (a) and $0.7 \mathrm{~mm}(\mathrm{~b}): 1-T=20^{\circ} \mathrm{C}, 2-80{ }^{\circ} \mathrm{C}$.

Fig. 12. Normal-hemispherical reflectance of an optically thick water mist layer in the submillimeter range at wavelengths $0.5 \mathrm{~mm}$ (a) and $0.7 \mathrm{~mm}(\mathrm{~b}): 1-T=20^{\circ} \mathrm{C}, 2-80^{\circ} \mathrm{C}$. 

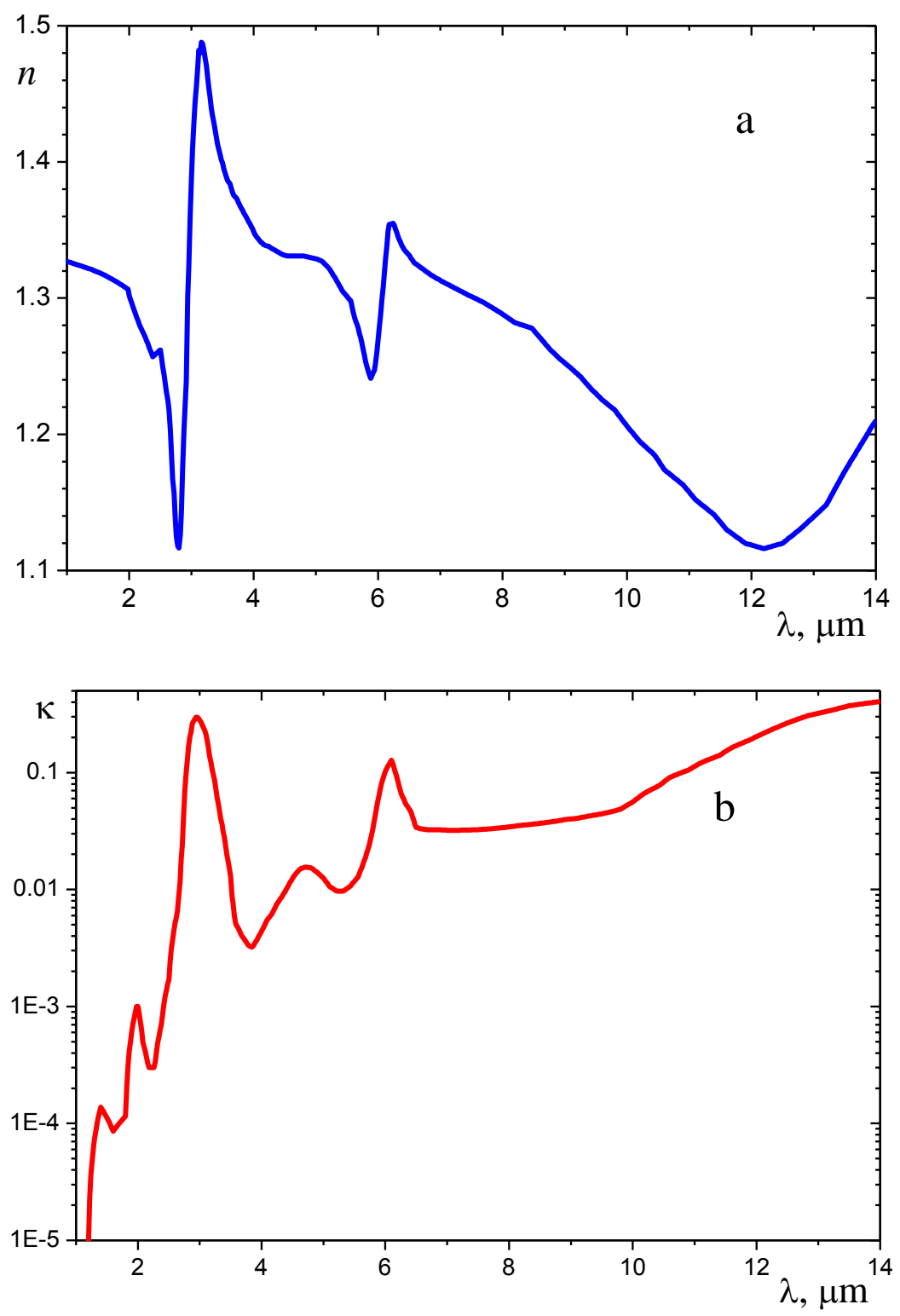

Figure 1. 


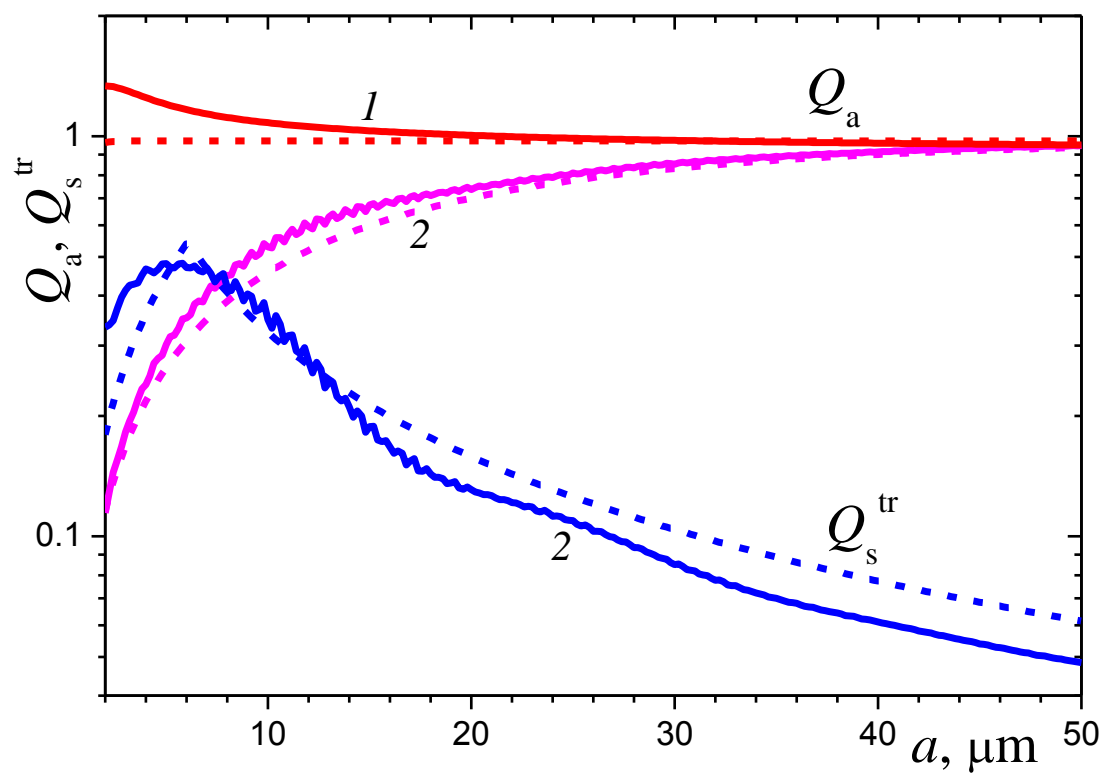

Figure 2 


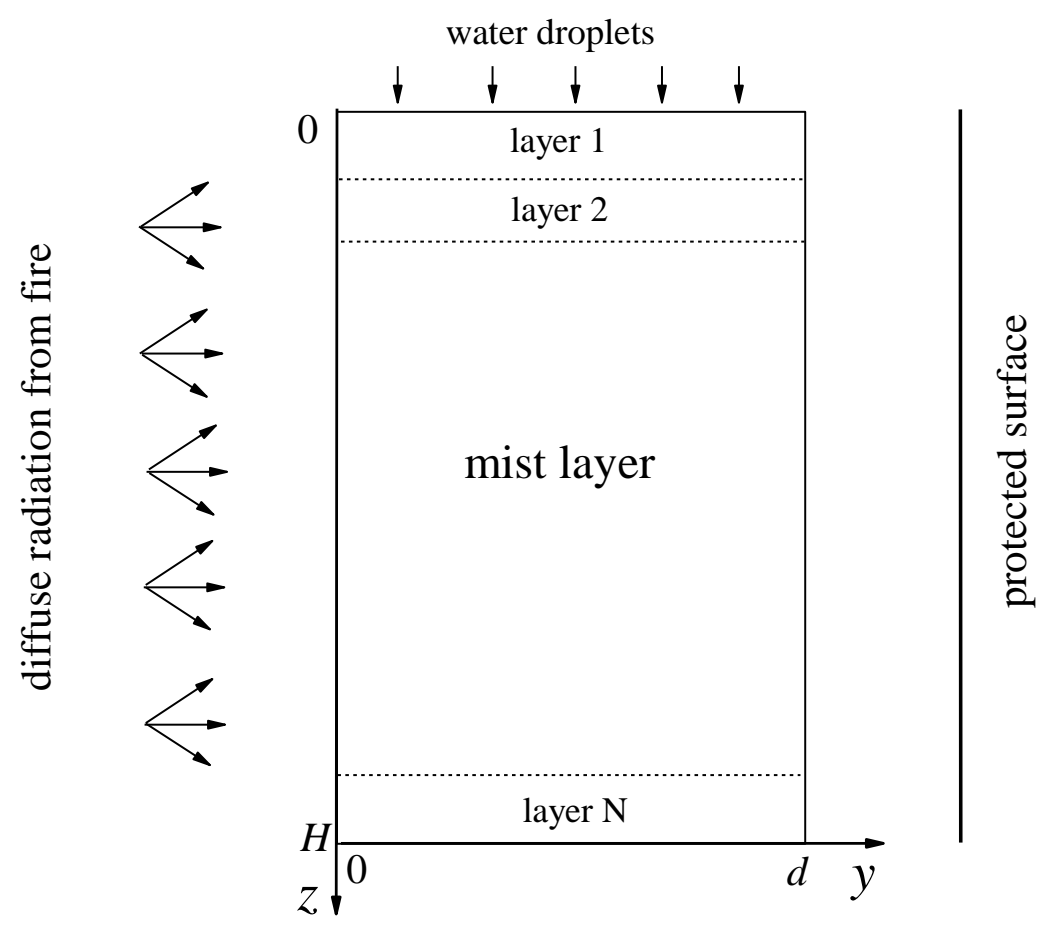

Figure 3 

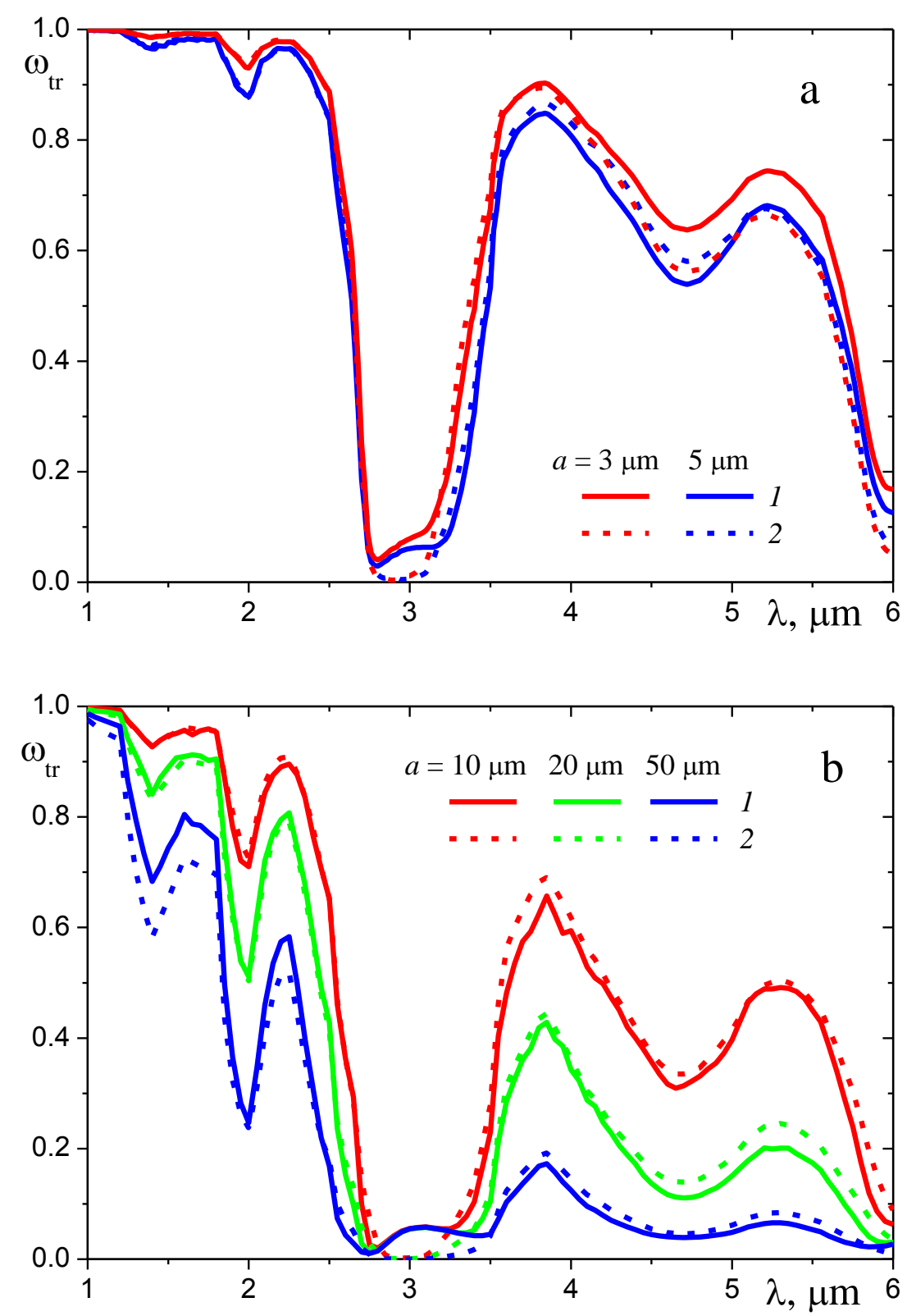

Figure 4 


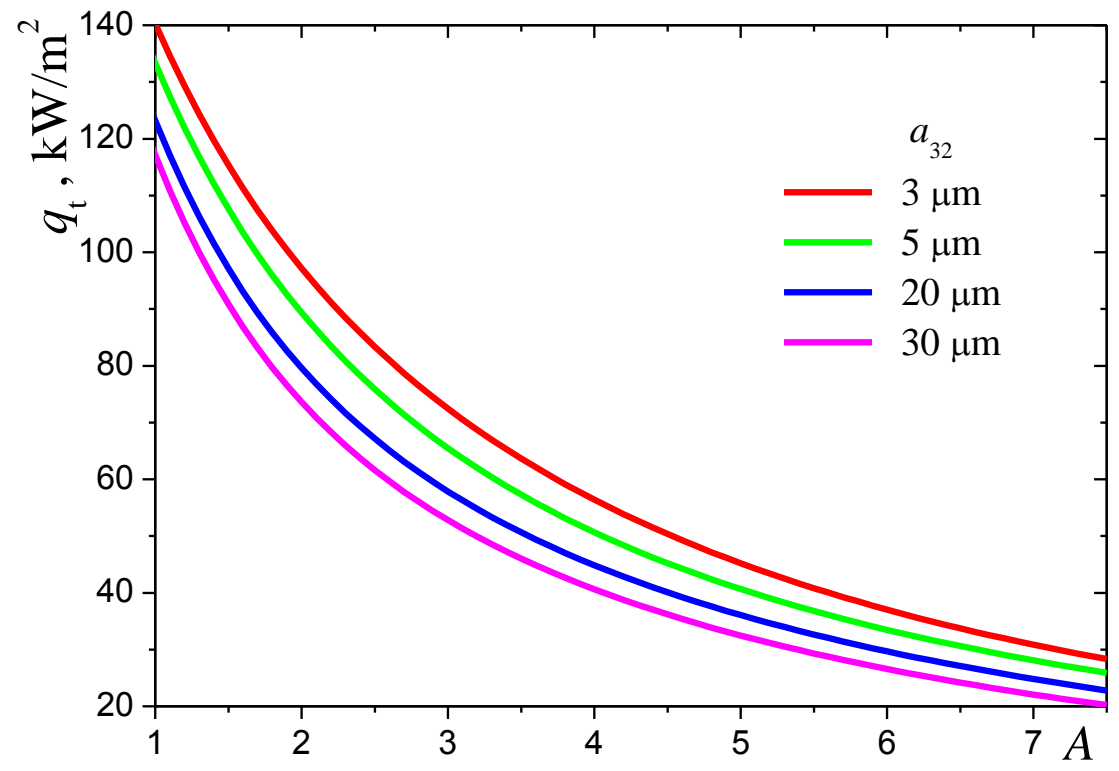

Figure 5 

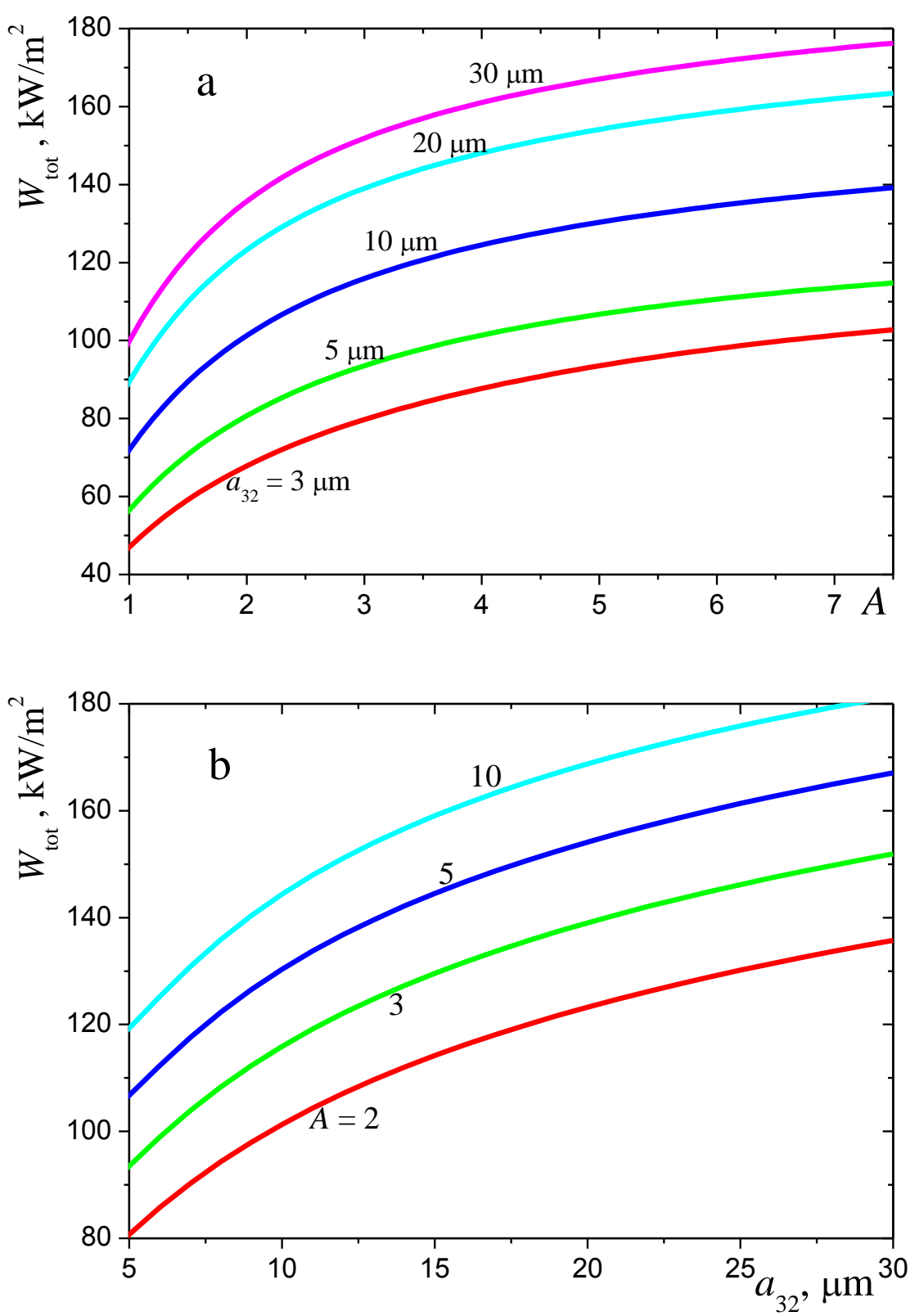

Figure 6 


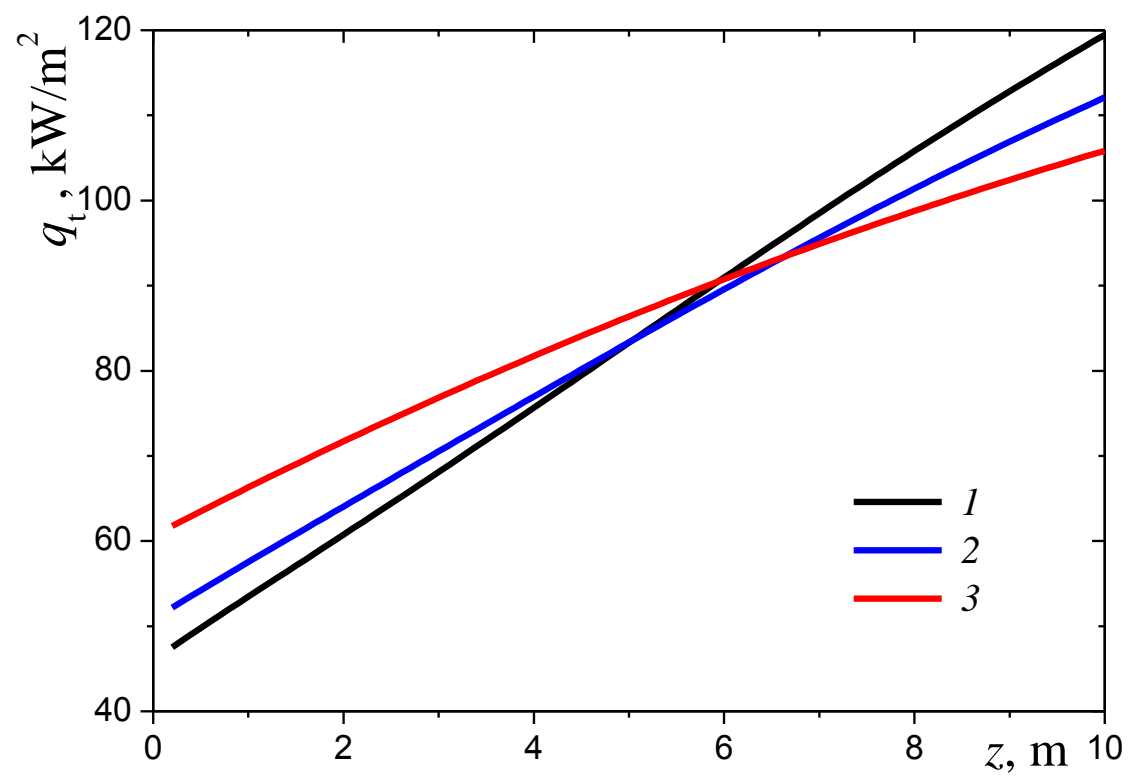

Figure 7 

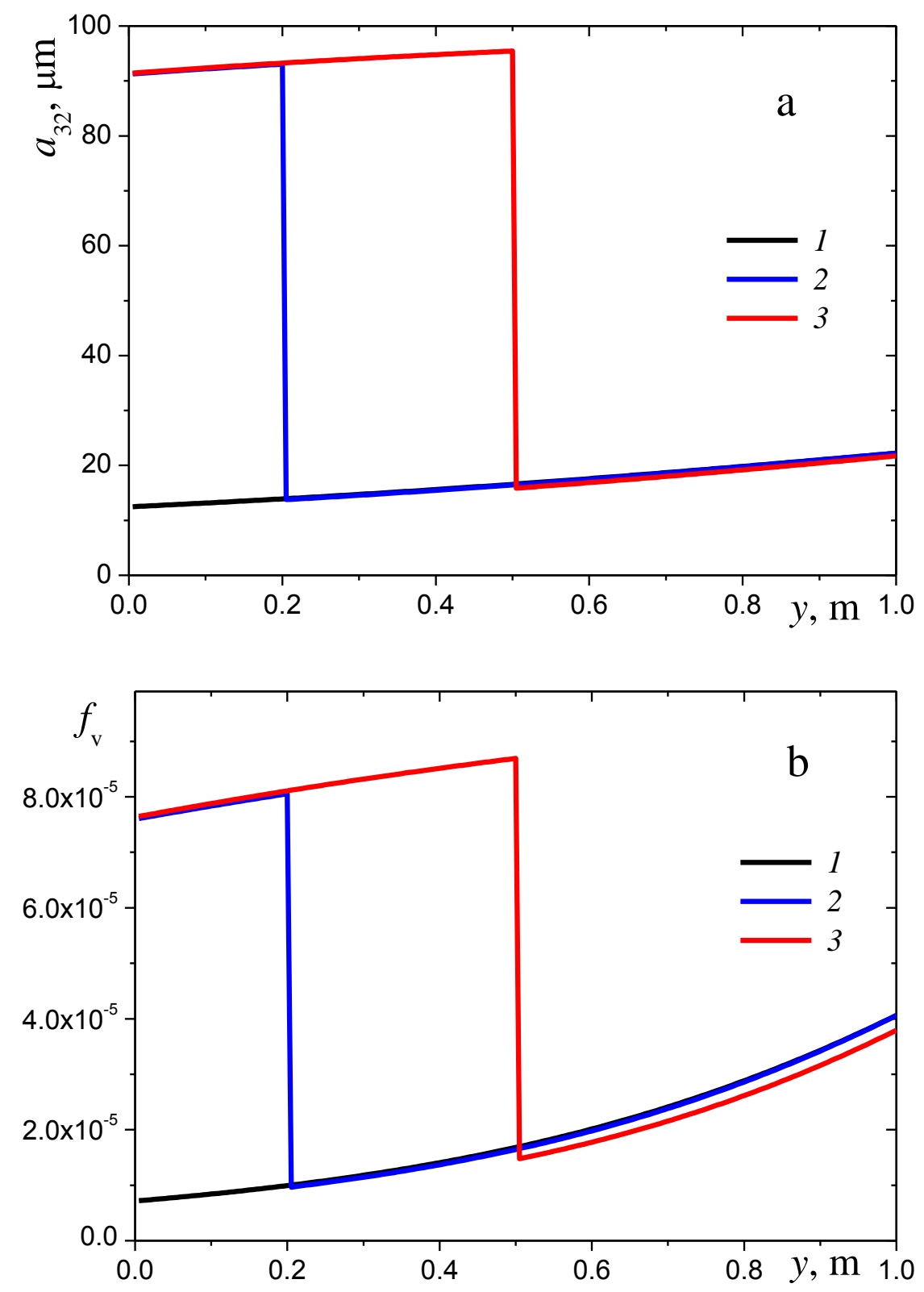

Figure 8 

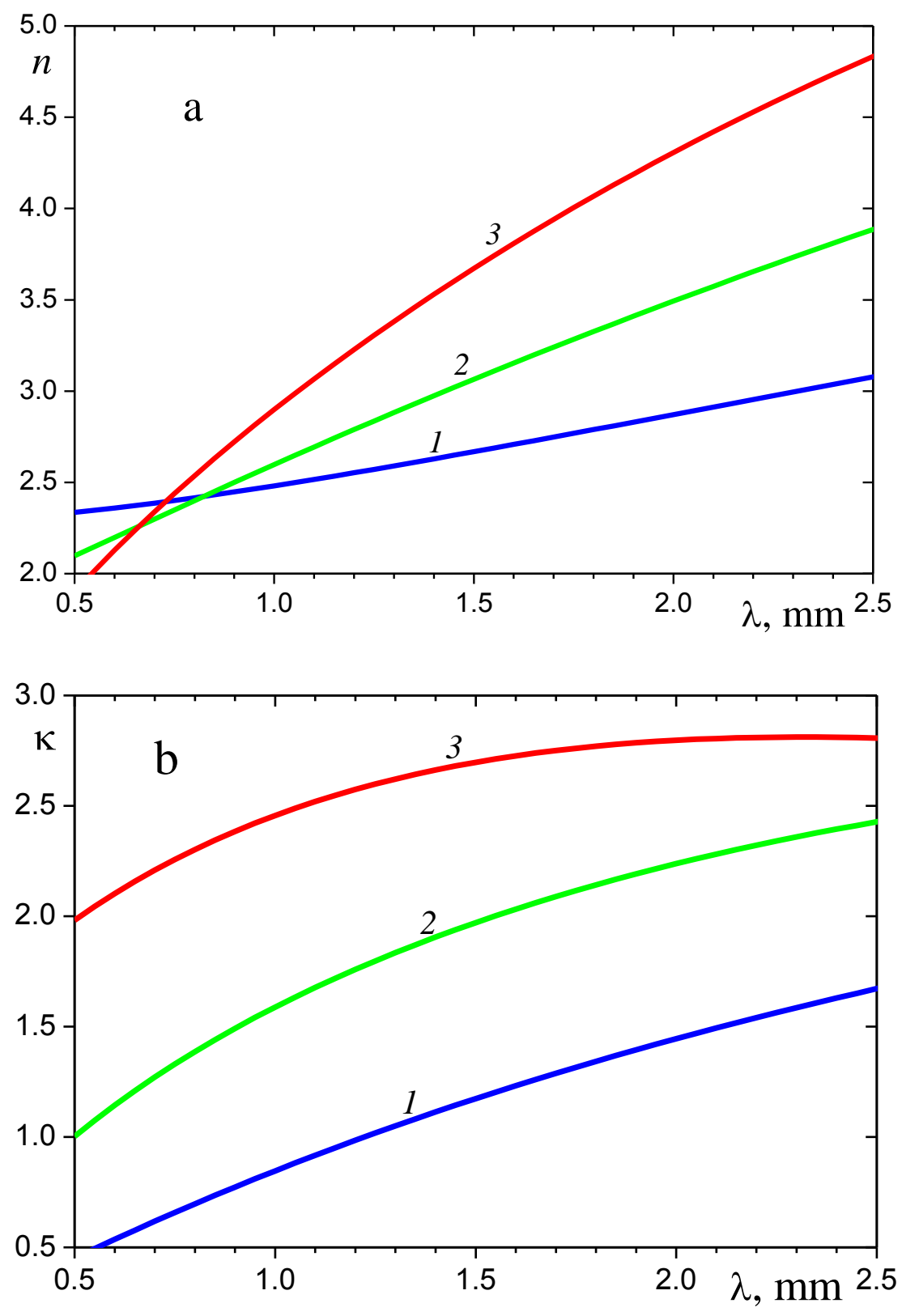

Figure 9 

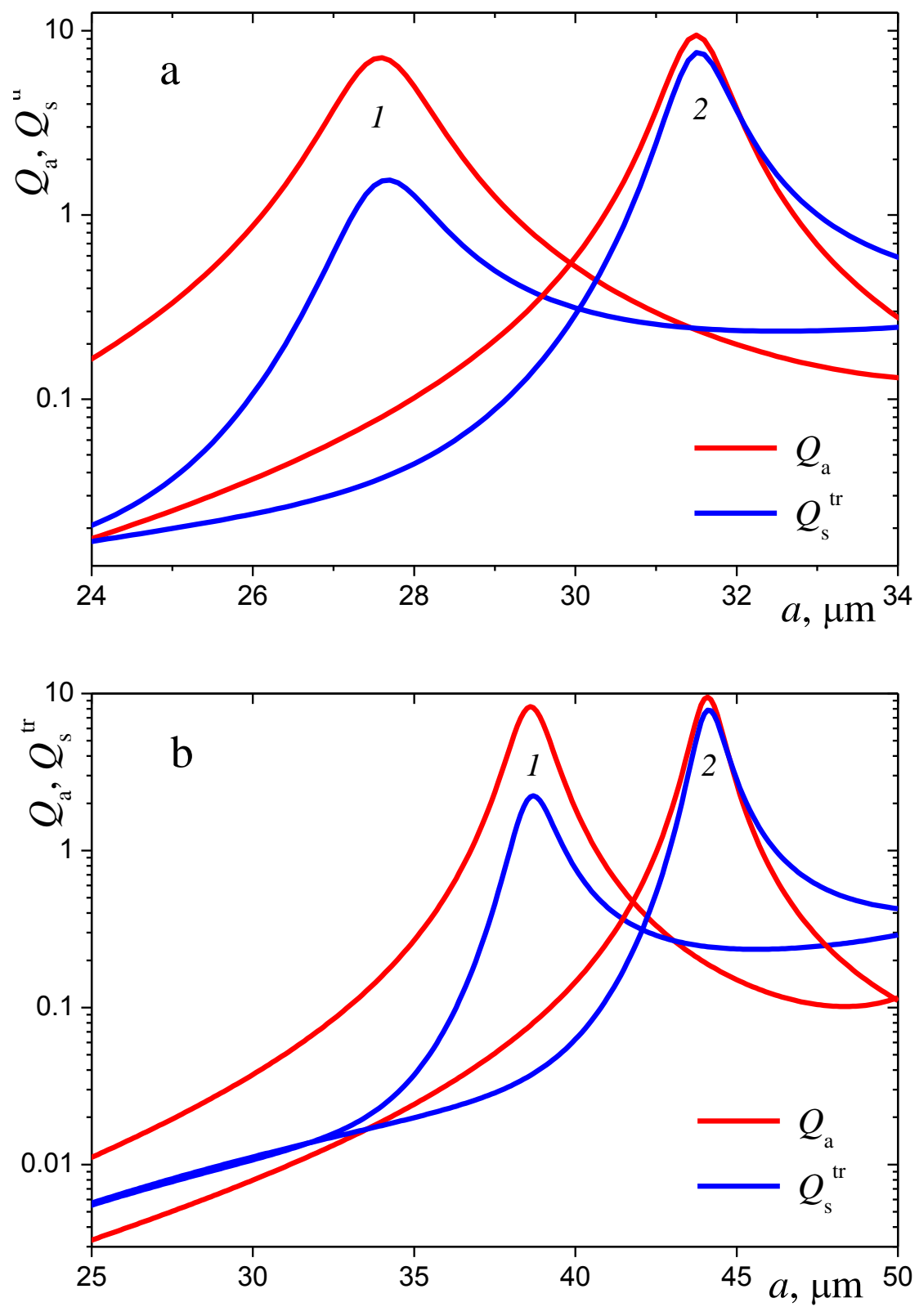

Figure 10 

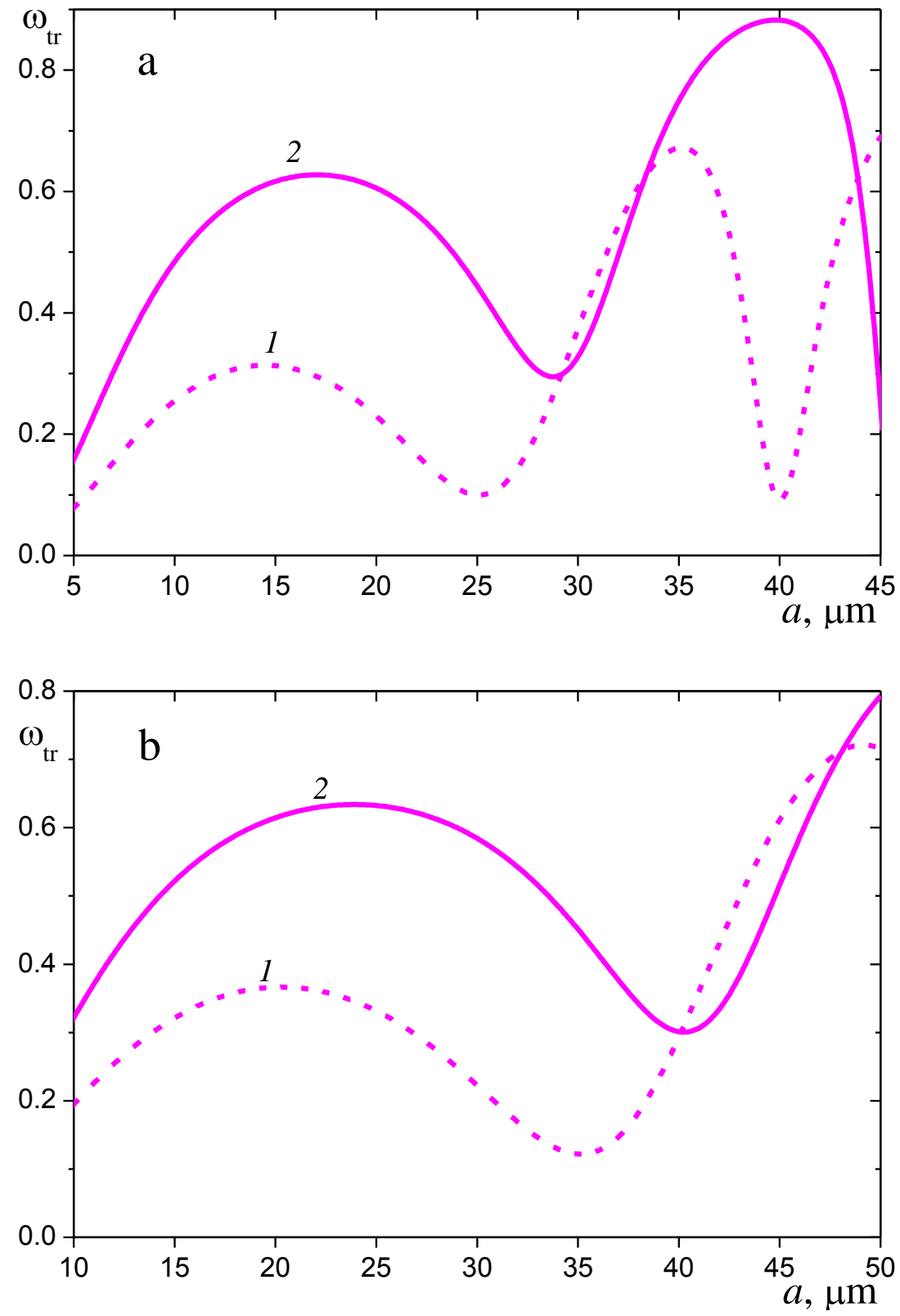

Figure 11 

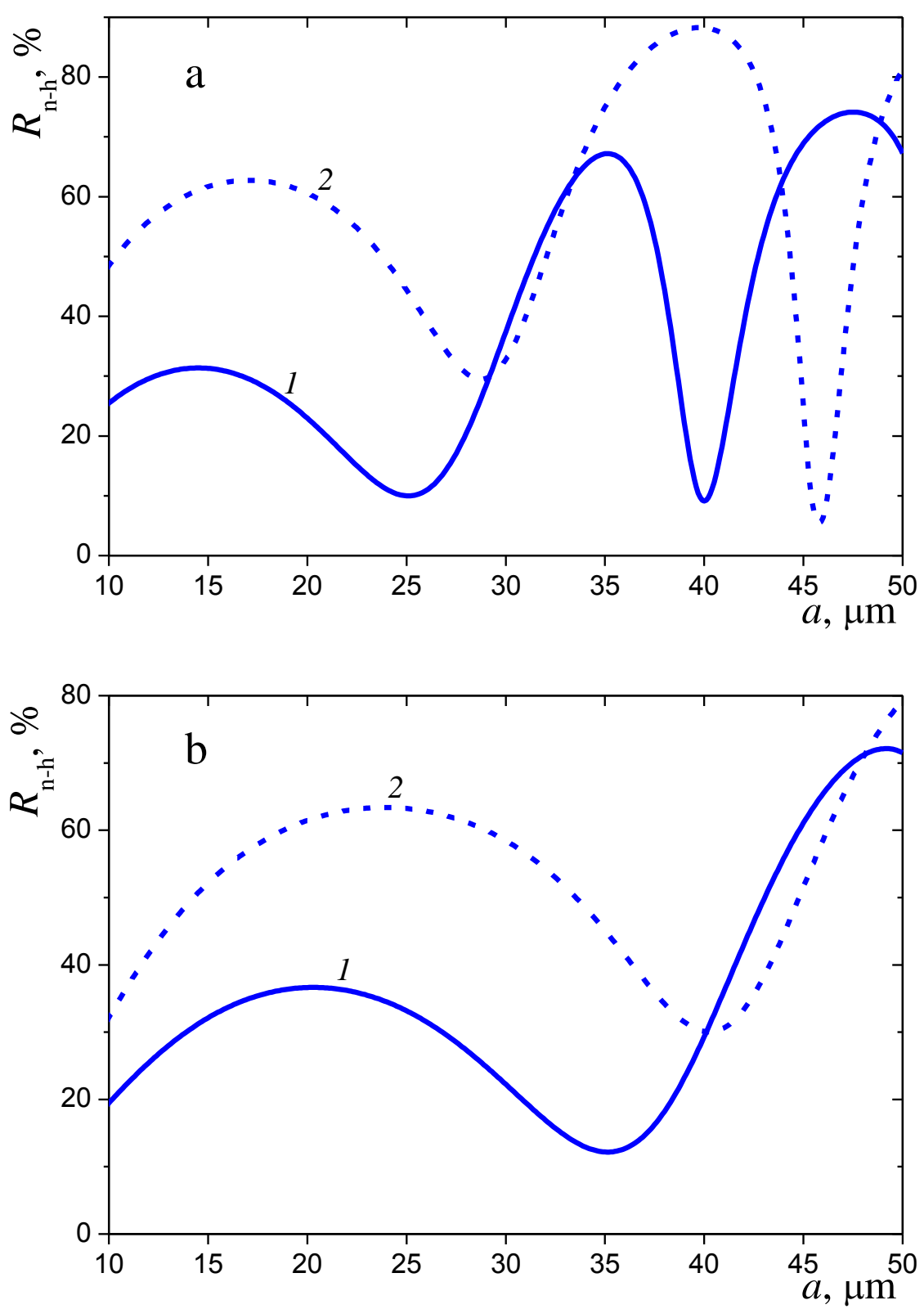

Figure 12 